\title{
Stresses and orientational order in shearing flows of granular liquid crystals
}

\author{
Diego Berzi, ${ }^{1}$ Nha Thai-Quang, ${ }^{2}$ Yu Guo, ${ }^{2}$ and Jennifer Curtis ${ }^{2}$ \\ ${ }^{1}$ Department of Civil and Environmental Engineering, Politecnico di Milano, 20133 Milano, Italy \\ ${ }^{2}$ Department of Chemical Engineering, University of Florida, Gainesville, Florida 32611, USA
}

(Received 20 January 2016; published 22 April 2016)

\begin{abstract}
We perform discrete element simulations of homogeneous shearing of frictionless cylinders and show that the particles are characterized by orientational order and form a granular liquid crystal. For elongated and flat cylinders, the alignment is in the plane of shearing, while cylinders having an aspect ratio equal to 1 and 0.8 show no orientational order. We show that the particle pressure is insensitive to the cylinder aspect ratio and well predicted by the kinetic theory of granular gases, with a singularity in the radial distribution function at contact different from that for frictionless spheres. The numerical results quantitatively agree with physical experiments on different geometries. The particle shear stress is affected by orientational anisotropy. We postulate that, for frictionless cylinders, the viscosity is roughly due to the motion of the orientationally disordered fraction of the particles, and show that it is proportional, through the order parameter, to the expression of kinetic theory. Finally, we suggest that the orientational order is the result of the competing effects of the shear rate, which induces alignment, and the granular temperature, which ramdomizes.
\end{abstract}

DOI: 10.1103/PhysRevE.93.040901

\section{INTRODUCTION}

The behavior of liquid crystals composed of elongated molecules is rather well understood, and continuum theory, able to predict the alignment of the molecules and its consequence on the transport properties of the material, is available $[1,2]$. On the other hand, the flow of nonspherical, macroscopic particles-of interest in many geophysical and industrial applications - has been less investigated, and a continuum theory is still lacking. Intuitively, and experimentally verified [3], shearing flows of macroscopic nonspherical particles can develop orientational order, while maintaining positional disorder, even if no external field is applied. The particles form a granular liquid crystal, an intermediate state between a granular gas (positionally and orientationally disordered) and a granular solid crystal (positionally and orientationally ordered).

Kinetic theory $[4,5]$ was originally intended for predicting dilute to moderately dense flows of granular gases composed of inelastic hard spheres. Building a theory based on first principles for flows of more realistic particles has proved a difficult task. Hence, ad hoc, but physically sound, modifications of classic kinetic theory have been proposed to take into account velocity correlation among the particles at large solid volume fractions [6-12], particle surface friction [13,14], and finite stiffness [15]. Recently [16,17], discrete element simulations on simple shear flows of cylinders at different solid volume fractions have been performed to study the influence of length-to-diameter (aspect) ratio, coefficient of collisional restitution, surface friction, and stiffness on the stresses, highlighting differences and similarities with the predictions of the kinetic theory for spheres.

Here, we focus on simple shearing of cylinders in the absence of gravity and aim to describe in detail how order and alignment of the particles depend on the solid volume fraction and aspect ratio, and how this information can be incorporated into a continuum model based on kinetic theory to describe stresses in flows of granular liquid crystals.

\section{SIMULATIONS AND THEORY}

We have performed numerical simulations of flows of frictionless cylinders of mass density $\rho_{p}$, length $L$, and diameter $d$ using the discrete element model described in Refs. $[16,18,19]$. Snapshots of the simple shear flows at solid volume fraction $v=0.4$ and three different aspect ratios $L / d$, together with the adopted frame of reference, are depicted in Figs. 1(a)-1(c). The only component of the mean particle velocity is in the $x$ direction and is linearly distributed along $y$, being $\dot{\gamma}$ the shear rate. In all the simulations, we kept constant the normal coefficient of collisional restitution (negative ratio of post- to precollisional relative velocity between two colliding particles) $e=0.95$, the dimensionless Young's modulus $E /\left(\rho_{p} \dot{\gamma}^{2} d_{v}^{2}\right)=1.5 \times 10^{9}$, where $d_{v}$ is the diameter of the equivalent sphere, and the Poisson's ratio equal to 0.3 in the Hertzian contact model.

The orientation of a cylinder is described using the inclination angle $\alpha$ and the azimuthal angle $\beta$ [Fig. 2(a)], both limited, for symmetry reasons, to the range $\pm 90^{\circ}$. As for molecular liquid crystals, we use the order parameter $S$ to describe the degree of order in the particle alignment [21]. Granular gases and crystal solids are characterized by $S=0$ and $S=1$, respectively. Intermediate values indicate that we are in the presence of granular liquid crystals. Figure 2(b) shows that the order parameter usually increases with the solid volume fraction and the maximum between $L / d$ and $d / L$, as previously noticed $[3,17]$. Cylinders with aspect ratios equal to 0.8 and 1 are in the granular gas state for the entire range of solid volume fraction [Fig. 1(b)]. This is due to the fact that the particle projected area on the plane perpendicular to the flow is essentially independent of the particle alignment, so that there is no preferential orientation of the particles to minimize the particle contacts [17]. For the other aspect ratios, the flow is in the granular liquid crystal state. The cases $L / d=0.25, L / d=0.5$, and $L / d=6$ present a maximum in the order parameter at a given value of the solid volume fraction. This might be due to differences in the dependence on 


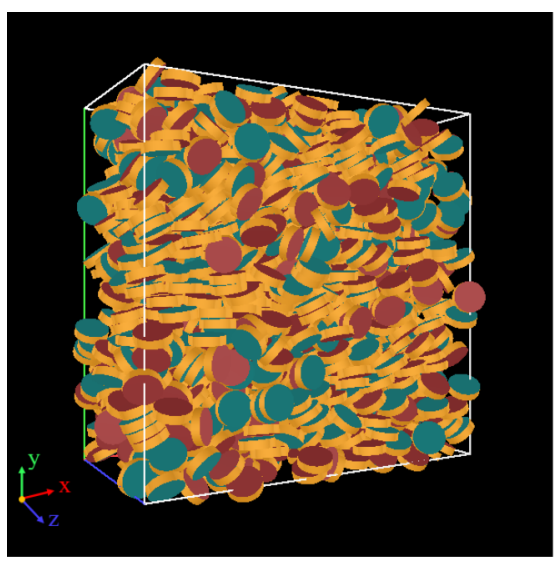

(a)

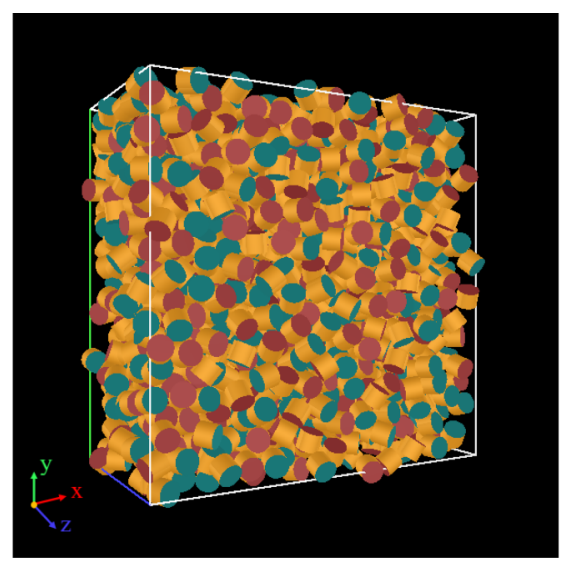

(b)

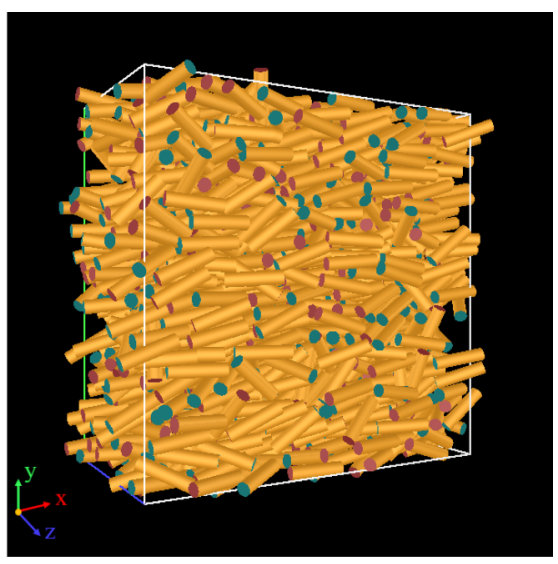

(c)

FIG. 1. Snapshots of the shear flows of cylinders at $v=0.4$ for (a) $L / d=0.25$, (b) $L / d=0.8$, and (c) $L / d=4 . x, y$, and $z$ are the flow, velocity gradient, and vorticity directions, respectively. Periodic boundary conditions along the $x$ and $z$ directions, and Lees-Edwards [20] boundary conditions along the $y$ direction have been employed.

$v$ for ordering in the planes of shear and vorticity. Figure 2(b) also indicates that there is no apparent first-order transition from an isotropic to a nematic state, i.e., a discontinuity in the order parameter, for shearing flows of cylinders, unlike the case of uniformly compressed hard nonspherical particles [22].

We have also measured the average alignment angle $\theta$ with respect to the flow direction [23]. Elongated cylinders ( $L / d$ larger than 1) align, on average, in the plane of shear [Fig. 1(c)] and the average alignment angle is less than $45^{\circ}$ and monotonically decreases with the order parameter [Fig. 2(c)]. The results of our simulations are in agreement with physical experiments on flows of glass cylinders and wooden pegs, characterized by $L / d$ in the range 3.3-5, in a split bottom shear cell [3]. As already shown [17], flat cylinders $(L / d$ equal to or less than 0.5) align again in the plane of shear [Fig. 2(a)], but the alignment angle is always larger than $45^{\circ}$ and increases with the order parameter [Fig. 2(c)].

The kinetic theory of granular gases provides an expression, in the case of randomly colliding spheres, for the particle pressure $p$, which has the form [5]

$$
\frac{p}{\rho_{p} T}=4 v^{2} g_{0}\left(\frac{1}{4 v g_{0}}+\frac{1+e}{2}\right),
$$

where $g_{0}$ is the radial distribution function at contactonly a function of the solid volume fraction-and $T$ is the granular temperature, mean square of the particle velocity fluctuations. Figure 3(a) depicts the dimensionless particle pressure obtained from the present simulations as a function of the solid volume fraction. The data collapse onto a single curve, irrespectively of the particle aspect ratio and, therefore, the orientational order. This may be understood given that both the pressure and the granular temperature are isotropic quantities, and hence insensitive to the anisotropy induced by the particle alignment. It indicates that kinetic theory is a good candidate as a continuum theory also for nonspherical particles. Experiments performed on gravity-driven collisional suspensions of plastic cylinders, of aspect ratio equal to 0.8 , in water [24,25] show the same collapse, and are in good agreement with the present numerical simulations [Fig. 3(b)]

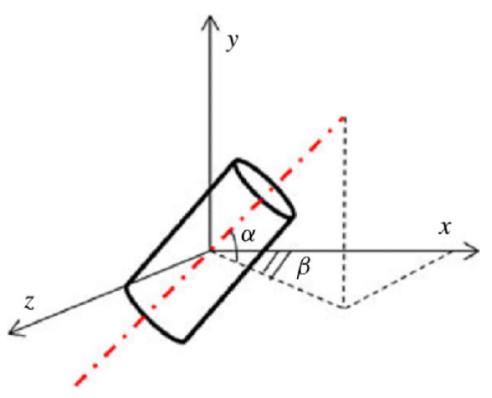

(a)

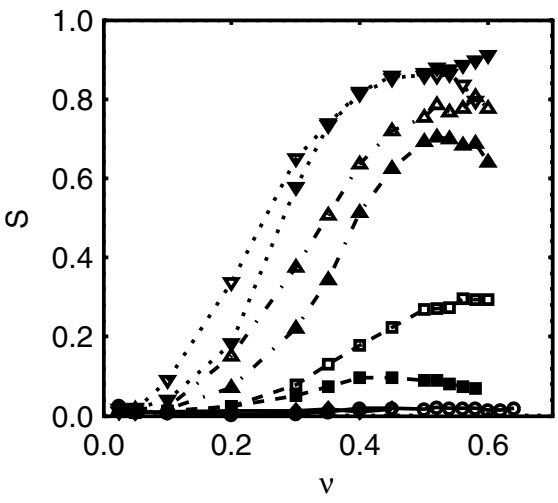

(b)

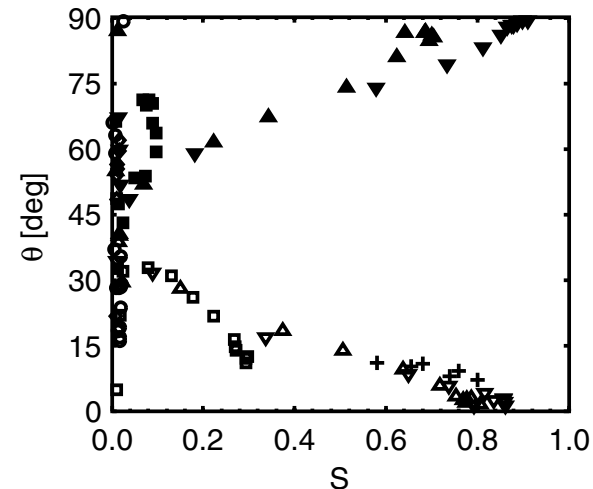

(c)

FIG. 2. (a) Cylinder orientation in terms of the two angles $\alpha$ and $\beta$. (b) Order parameter as a function of the solid volume fraction for $L / d=0.17$ (solid lower triangles), $L / d=0.25$ (solid upper triangles), $L / d=0.5$ (solid squares), $L / d=0.8$ (diamonds), $L / d=1$ (circles), $L / d=2$ (hollow squares), $L / d=4$ (hollow upper triangles), and $L / d=6$ (hollow lower triangles). (c) Average alignment angle as a function of the order parameter [same symbols as in Fig. 2(b)]. Also shown are the experimental values for glass cylinders and wooden pegs [3] (crosses). 


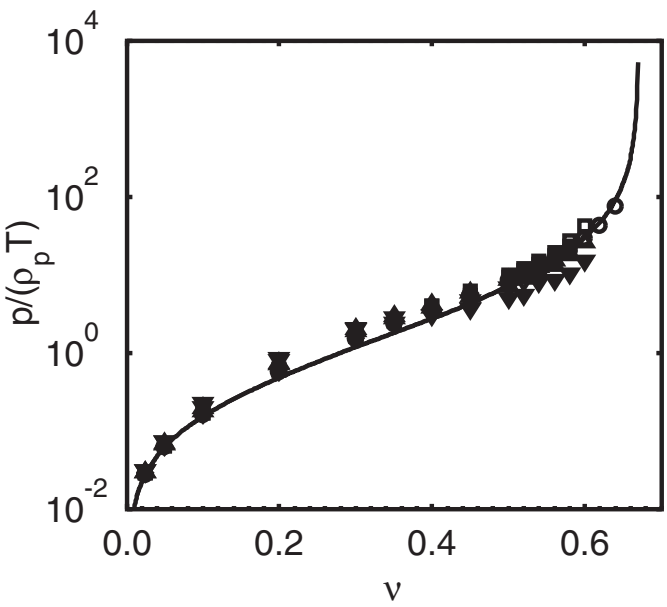

(a)

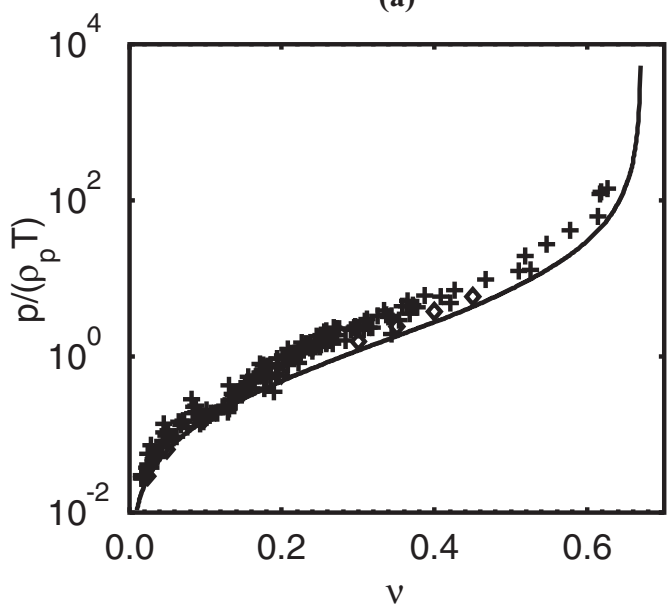

(b)

FIG. 3. Dimensionless pressure versus solid volume fraction obtained from the present numerical simulations [same symbols as in Fig. 2(b)] and experiments (crosses) on collisional suspensions of plastic cylinders in water. The solid line represents Eq. (1).

[26]. The experimental and numerical data can be satisfactorily reproduced with Eq. (1), if we use, as suggested by Vescovi et al. [12] and valid for frictionless spheres with $e$ less than or equal to 0.95 , the radial distribution function at contact of Carnahan and Starling [27]:

$$
g_{0}=\frac{2-v}{2(1-v)^{3}}
$$

if $v \leqslant 0.4$; and

$g_{0}=\left[1-\left(\frac{v-0.4}{v_{c}-0.4}\right)^{2}\right] \frac{2-v}{2(1-v)^{3}}+\left(\frac{v-0.4}{v_{c}-0.4}\right)^{2} \frac{2}{v_{c}-v}$,

if $v>0.4$. In Eq. (3), $v_{c}$ represents the critical value of the solid volume fraction at which $g_{0}$ is singular, which is only a function of the surface friction for spheres [14]. In the case of frictionless cylinders, $v_{c}=0.67$ allows good agreement with the data (Fig. 3).

Kinetic theory [5] predicts also that the ratio of the particle shear stress to the shear rate, i.e., the viscosity $\eta$, in a granular

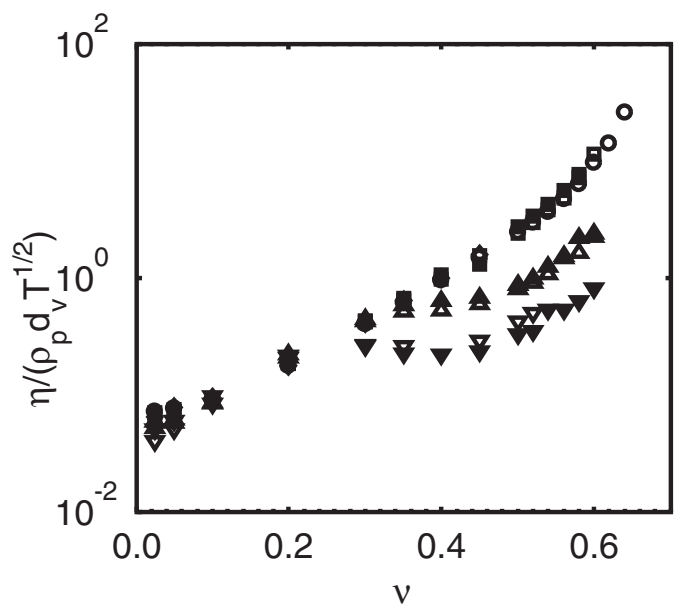

(a)

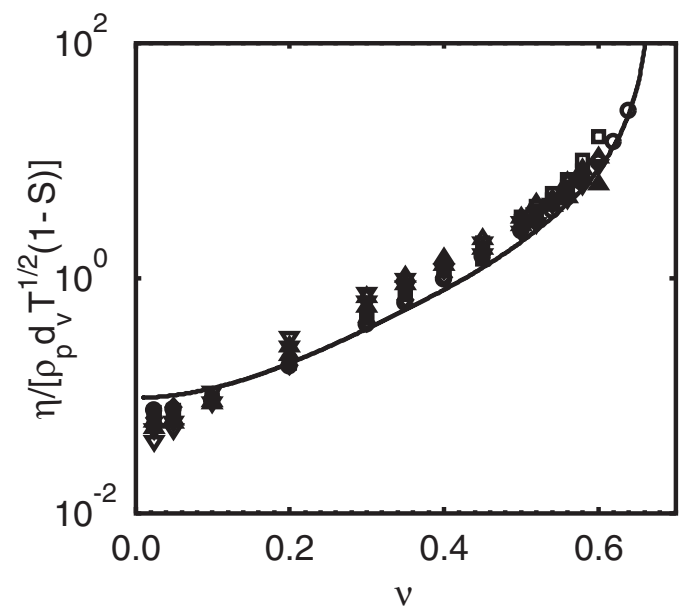

(b)

FIG. 4. (a) Dimensionless viscosity versus solid volume fraction obtained from the present numerical simulations [same symbols as in Fig. 2(b)]. (b) Same as in Fig. 4(a), but the dimensionless viscosity is scaled with $1-S$. The solid line represents Eq. (5).

gas is given by

$$
\frac{\eta}{\rho_{p} d_{v} T^{1 / 2}}=\frac{8 J}{5 \pi^{1 / 2}} v^{2} g_{0}
$$

where $J$ is a known function of the coefficient of restitution and the solid volume fraction [28]. Figure 4(a) shows the dimensionless viscosity as a function of the solid volume fraction obtained from our numerical simulations. As previously noticed $[3,16,17]$, the particle alignment in the plane of shear causes the viscosity to be greatly reduced with respect to the isotropic case, $L / d=1$. Unlike the pressure, the shear stress is sensitive to the flow anisotropy, so that the numerical data do not collapse onto a single curve and Eq. (4) of kinetic theory does not hold. We now imagine that the viscosity of a granular liquid crystal has two components, associated with the orientationally random and ordered motion of the particles, whose relative importance is naturally measured through the order parameter, by $1-S$ and $S$, respectively. Frictionless cylinders, perfectly aligned with their axes along the flow direction, can slide over each other without any resistance. Hence, as a first approximation, we assume that only the 


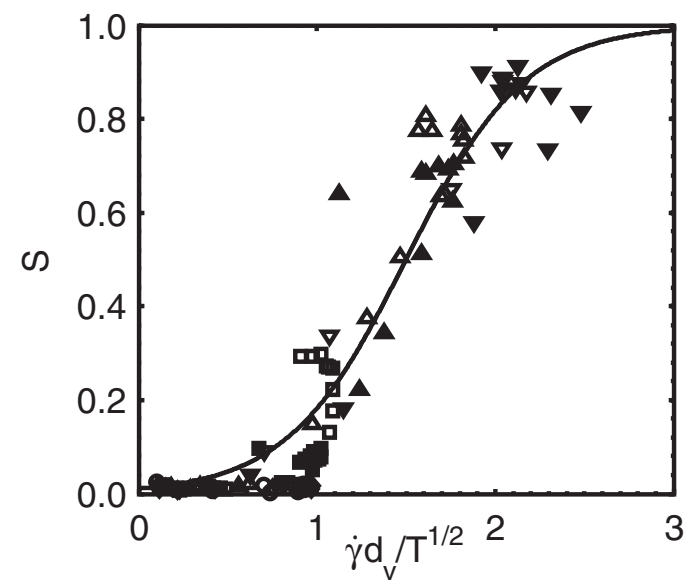

FIG. 5. Order parameter as a function of the ratio of the shear rate to the square root of the granular temperature [same symbols as in Fig. 2(b)]. The solid line is a tentative fitting using the logistic function $S=1 /\left[1+\exp \left(6-4 \dot{\gamma} d_{v} / T^{1 / 2}\right)\right]$.

orientationally random motion of the cylinders contributes to the viscosity. If the component of the viscosity associated with the orientationally random motion is given by kinetic theory, we obtain

$$
\frac{\eta}{\rho_{p} d_{v} T^{1 / 2}} \simeq(1-S) \frac{8 J}{5 \pi^{1 / 2}} v^{2} g_{0} .
$$

When the dimensionless viscosity, divided by $1-S$, is plotted against the solid volume fraction, we obtain a collapse of the data from the present numerical simulations [Fig. 4(b)]. Equation (5), with $g_{0}$ once again given by Eqs. (2) and (3) and $v_{c}=0.67$, is in excellent agreement with the measurements when $L / d=1$ and 0.8 (no orientational order), while it slightly underestimates the viscosity for the other aspect ratios when $v$ exceeds 0.2 , due to the imperfect alignment of the particles along the flow direction. When $v$ is less than 0.1 , there is no orientational order [Fig. 2(b)]: there, the lack of agreement of the simulations with the kinetic theory of Garzó and Dufty [5] is probably due to anisotropy in the particle velocity distribution function [29].

The order parameter is a key quantity in the expression of the viscosity and, therefore, the particle shear stress. Hence, it must be determined from a suitable balance equation in the framework of a continuum theory for predicting the flow of granular liquid crystals. Following a heuristic argument-originally applied to the velocity correlation between the particles at solid volume fraction larger than the freezing value $[7,11,30]$-we assume that the order parameter results from a competition between the alignment induced by the shear rate and randomization due to the velocity fluctuations, i.e., the granular temperature. Hence, at equilibrium, the order parameter should be an increasing function of the dimensionless quantity $\dot{\gamma} d_{v} / T^{1 / 2}$. Figure 5 shows the satisfactory collapse of the measurements obtained from the present numerical simulations. It is evident that there is no orientational order if the ratio $\dot{\gamma} d_{v} / T^{1 / 2}$ is less than 1. When $\dot{\gamma} d_{v} / T^{1 / 2}$ exceeds 1 , the order parameter progressively increases and would eventually approximate 1 for large enough shear rate and/or low enough granular temperature.

\section{CONCLUSIONS}

In this work, we have shown that cylindrical particles in shearing flows are, generally, in the granular liquid crystalline state, whose orientational order usually increases with the solid volume fraction, with no apparent first-order phase transition. Elongated and flat cylinders align themselves in the plane of shear, and form an angle with the flow direction which is less and greater than $45^{\circ}$, respectively. Cylinders having aspect ratios equal to 0.8 and 1 show no orientational order. We have demonstrated that the particle pressure scales with the granular temperature and can be predicted using the kinetic theory of granular gases, if the singularity in the radial distribution function at contact is taken to be 0.67 . We have suggested that the viscosity has one component associated with the orientationally random motion of the particles, given by the expression of kinetic theory multiplied by $1-S$, and a second component associated with the orientationally ordered motion of the particles. We have finally suggested that the order parameter is a consequence of the competition between the ordering of the shear rate and the disordering of the velocity fluctuations. Although the present numerical simulations have been performed on the almost ideal case of homogeneous shearing of frictionless cylinders, the quantitative agreement with physical experiments carried out in more complicated geometries, even in the presence of an interstitial fluid, supports the generality of our findings.
[1] J. T. Jenkins, Ann. Rev. Fluid. Mech. 10, 197 (1978).

[2] F. M. Leslie, Adv. Liq. Cryst. 4, 1 (1979).

[3] T. Börzsönyi, B. Szabó, G. Törös, S. Wegner, J. Török, E. Somfai, T. Bien, and R. Stannarius, Phys. Rev. Lett. 108, 228302 (2012).

[4] J. Jenkins and S. Savage, J. Fluid. Mech. 130, 187 (1983).

[5] V. Garzó and J. W. Dufty, Phys. Rev. E 59, 5895 (1999).

[6] N. Mitarai and H. Nakanishi, Phys. Rev. E 75, 031305 (2007).

[7] J. T. Jenkins, Granular Matter 10, 47 (2007).
[8] V. Kumaran, J. Fluid Mech. 632, 145 (2009).

[9] J. Jenkins and D. Berzi, Granular Matter 12, 151 (2010).

[10] J. Jenkins and D. Berzi, Granular Matter 14, 79 (2012).

[11] D. Berzi, Acta Mech. 225, 2191 (2014).

[12] D. Vescovi, D. Berzi, P. Richard, and N. Brodu, Phys. Fluids 26, 053305 (2014).

[13] J. Jenkins and C. Zhang, Phys. Fluids 14, 1228 (2002).

[14] D. Berzi and D. Vescovi, Phys. Fluids 27, 013302 (2015).

[15] D. Berzi and J. Jenkins, Soft Matter 11, 4799 (2015). 
[16] Y. Guo, C. Wassgren, W. Ketterhagen, B. Hancock, B. James, and J. Curtis, J. Fluid Mech. 713, 1 (2012).

[17] Y. Guo, C. Wassgren, B. Hancock, W. Ketterhagen, and J. Curtis, Phys. Fluids 25, 063304 (2013).

[18] M. Kodam, M. Bharadwaj, J. Curtis, B. Hancock, and C. Wassgren, Chem. Eng. Sci. 65, 5852 (2010).

[19] Y. Guo, C. Wassgren, W. Ketterhagen, B. Hancock, and J. Curtis, Powder Technol. 228, 193 (2012).

[20] A. W. Lees and S. F. Edwards, J. Phys. C: Solid State Phys. 5, 1921 (1972).

[21] We calculate $S$ as the largest eigenvalue of the symmetric traceless tensor $Q_{i j}=3 /(2 N) \sum_{n-1}^{N}\left(\ell_{i}^{n} \ell_{j}^{n}-\delta_{i j} / 3\right)$, where $\ell^{n}$ is the unit vector along the axis of the $n$th cylindrical particle and the sum is over all the $N$ particles of the system [3,31].

[22] M. P. Allen and M. R. Wilson, J. Comput.-Aided Mol. Des. 3, 335 (1989).

[23] It corresponds to the deviation of the largest main axis of $\mathbf{Q}$ from the $x$ direction.
[24] H. Capart and L. Fraccarollo, Geophys. Res. Lett 38, L20402 (2011).

[25] D. Berzi and L. Fraccarollo, Phys. Rev. Lett. 115, 194501 (2015).

[26] Berzi and Fraccarollo [25] calculated the normal stresses in the direction perpendicular to the flow from the integration of the profiles of solid volume fraction. The experimental pressure in Fig. 3(b) has been estimated by multiplying the normal stress by the measured ratio of the (isotropic) granular temperature to the mean square of the velocity fluctuations in the direction perpendicular to the flow.

[27] N. F. Carnahan and K. E. Starling, J. Chem. Phys. 51, 635 (1969).

[28] $J=(1+e) / 2+(\pi / 32)\left[5+2(1+e)(3 e-1) v g_{0}\right][5+4(1+e)$ $\left.v g_{0}\right] /\left\{\left[24-6(1-e)^{2}-5\left(1-e^{2}\right)\right] v^{2} g_{0}^{2}\right\}$, from, e.g., Jenkins and Berzi [9].

[29] J. T. Jenkins and M. W. Richman, J. Fluid Mech. 192, 313 (1988).

[30] J. T. Jenkins, Phys. Fluids 18, 103307 (2006).

[31] S. Wegner, T. Börzsönyi, T. Bien, G. Rose, and R. Stannarius, Soft Matter 8, 10950 (2012). 